\title{
The Hubble Space Telescope UV Legacy Survey of Galactic Globular Clusters. XVIII. Proper-motion Kinematics of Multiple Stellar Populations in the Core Regions of NGC 6352
}

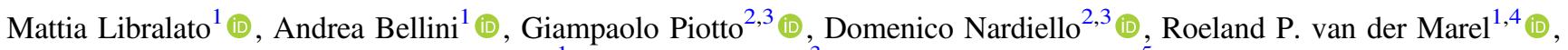 \\ Jay Anderson ${ }^{1}$ (D), Luigi R. Bedin ${ }^{3}$ (D), and Enrico Vesperini ${ }^{5}$ (D) \\ ${ }^{1}$ Space Telescope Science Institute 3700 San Martin Drive, Baltimore, MD 21218, USA; libra@stsci.edu \\ ${ }^{2}$ Dipartimento di Fisica e Astronomia, Università di Padova, Vicolo dell'Osservatorio 3, Padova, I-35122, Italy \\ ${ }^{3}$ INAF-Osservatorio Astronomico di Padova, Vicolo dell'Osservatorio 5, Padova, I-35122, Italy \\ ${ }^{4}$ Center for Astrophysical Sciences, Department of Physics \& Astronomy, Johns Hopkins University, Baltimore, MD 21218, USA \\ ${ }^{5}$ Department of Astronomy, Indiana University, Bloomington, IN 47405, USA \\ Received 2018 December 24; revised 2019 February 5; accepted 2019 February 6; published 2019 March 11
}

\begin{abstract}
We present the analysis of the radial distributions and kinematic properties of the multiple stellar populations (mPOPs) hosted in the globular cluster (GC) NGC 6352 as part of the Hubble Space Telescope "UV Legacy Survey of Galactic Globular Clusters" program. NGC 6352 is one of the few GCs for which the mPOP tagging in appropriate color-magnitude diagrams is clear in all evolutionary sequences. We computed high-precision stellar proper motions for the stars from the cluster's core out to $75 \operatorname{arcsec}(\sim 1.5$ core radii, or $\sim 0.6$ half-light radii). We find that, in the region explored, first- and second-generation stars share the same radial distribution and kinematic properties. Velocity dispersions, anisotropy radial profiles, differential rotation, and level of energy equipartition, all suggest that NGC 6352 is probably in an advanced evolutionary stage, and any possible difference in the structural and kinematic properties of its mPOPs have been erased by dynamical processes in the core of the cluster. We also provide an estimate of the mass of blue stragglers and of main-sequence binaries through kinematics alone. In general, in order to build a complete dynamical picture of this and other GCs, it will be essential to extend the analyses presented in this paper to the GCs' outer regions where some memories of the initial differences in the mPOP properties, and those imprinted by dynamical processes, might still be present.
\end{abstract}

Key words: globular clusters: individual (NGC 6352) - proper motions - stars: kinematics and dynamics - stars: Population II - techniques: photometric

\section{Introduction}

The Hubble Space Telescope (HST) "UV Legacy Survey of Galactic Globular Clusters" program (GO-13297, PI: Piotto; see Piotto et al. 2015) has started a systematic, photometric analysis of the multiple stellar populations (mPOPs) hosted in globular clusters (GCs). We now know that essentially all GCs studied with the proper tools show the presence of mPOPs, and that the mPOPs found in different GCs are characterized by a wide variety of properties.

After the initial studies focused on the identification of mPOPs, we worked on the full characterization of the mPOP properties. We have analyzed most of the photometric pieces of information contained in this Treasury survey, but the wealth of information contained in this data set is still far from being completely explored and revealed. We have begun to study the kinematic properties of the mPOPs thanks to state-of-the-art proper motions (PMs) in light of the recent work in this research field (Anderson \& van der Marel 2010; Richer et al. 2013; Bellini et al. 2015, 2018; Libralato et al. 2018; Milone et al. 2018).

In this work, we continue the investigation of the GC NGC 6352 started by Nardiello et al. (2015). NGC 6352 is a metal-rich $([\mathrm{Fe} / \mathrm{H}]=-0.67$, Carretta \& Gratton 1997) GC in the Bulge direction with a mass of $6.1 \times 10^{4} M_{\odot}$ (Baumgardt et al. 2019). Nardiello et al. (2015) demonstrated the presence of two populations clearly distinguishable in the red-giant branch (RGB), subgiant branch (SGB), main sequence (MS), asymptotic giant branch (AGB), and horizontal branch (HB) with UV-optical color-magnitude diagrams (CMDs). The firstgeneration $(1 \mathrm{G})$ population (hereafter, $\mathrm{POPa}$ ) has a primordial chemical composition, while the second-generation $(2 \mathrm{G})$ population $(\mathrm{POPb})$ is almost coeval $(\triangle \mathrm{Age}=10 \pm 120 \mathrm{Myr})$, slightly enhanced in $\mathrm{He}(\Delta Y=0.029 \pm 0.006)$, and in $\mathrm{Na}$ (e.g., Feltzing et al. 2009).

In this paper, we focus our attention on the structural and kinematic properties of the mPOPs in this cluster. We first compute HST-based, high-precision PMs to select members of NGC 6352 among the multitude of (Bulge and Disk) field stars. We then separate $\mathrm{POPa}$ and $\mathrm{POPb}$, and study their radial distributions and internal kinematics.

\section{Data Sets and Reduction}

We made use of _flc exposures ${ }^{6}$ taken with the Wide-Field Channel (WFC) of the Advanced Camera for Survey (ACS) and with the Ultraviolet-VISible (UVIS) channel of the Wide-Field Camera 3 (WFC3). The complete list of observations $^{7}$ is shown in Table 1.

The data reduction is a combination of first- and a secondpass photometric stages, and was performed as in Bellini et al. (2018) and Libralato et al. (2018). We refer to these papers for an extensive description of the work flow.

\footnotetext{
6 _flt images pipeline corrected for the charge-transfer-efficiency defects as described in Anderson \& Bedin (2010).

7 doi:10.17909/t9-m7g1-8a93
} 
Table 1

List of Observations of NGC 6352 Used in This Paper

\begin{tabular}{|c|c|c|c|c|c|}
\hline$\overline{\mathrm{GO}}$ & PI & Instrument/Camera & Filter & $N \times$ Exp. Time & Epoch \\
\hline 10775 & & & F814W & $4 \times 150 \mathrm{~s}, 1 \times 7 \mathrm{~s}$ & \\
\hline \multirow[t]{2}{*}{12746} & Kong & ACS/WFC & F625W & $2 \times 150 \mathrm{~s}$ & $2012 \mathrm{Feb}$ \\
\hline & & WFC3/UVIS & F336W & $5 \times 400 \mathrm{~s}, 1 \times 410 \mathrm{~s}$ & \\
\hline \multirow[t]{4}{*}{13297} & Piotto & WFC3/UVIS & F275W & $2 \times 706 s$ & 2013 Aug \\
\hline & & & & $2 \times 800 \mathrm{~s}$ & 2014 May \\
\hline & & & F438W & $1 \times 58 \mathrm{~s}$ & 2013 Aug \\
\hline & & & & $1 \times 72 \mathrm{~s}$ & 2014 May \\
\hline
\end{tabular}

The first-pass photometry allows us to detect the brightest, most isolated sources in each exposure in a single finding wave, and measure position and flux of these objects via pointspread-function (PSF) fit. The publicly available, spatially variable $H S T$ library PSFs $^{8}$ are tailored to each exposure, and stellar positions are corrected for geometric distortion using the state-of-the-art corrections available for HST detectors (Anderson \& King 2006; Bellini \& Bedin 2009; Bellini et al. 2011). Positions and fluxes are then used to build a common referenceframe system.

The second-pass photometry employs all images at once to enhance the contribution of faint sources. All close-by neighbors are subtracted from each image prior to estimate position and flux of a given source, thus improving the measurements in crowded regions.

The main differences with Libralato et al. (2018) are that (i) we used the Gaia Data Release 2 (DR2; Gaia Collaboration et al. 2016, 2018) to set up orientation ( $X$ and $Y$ axes toward

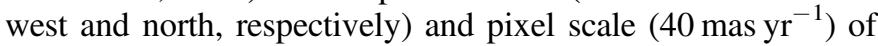
our reference-frame system, and (ii) we run the second-pass photometry tool separately for the three data sets (GO-10775, GO-12746, and GO-13297) as done in Bellini et al. (2018), so as to measure stars that might have moved by more than 1 pixel from one epoch to another.

We calibrated our photometry on to the Vega-mag system following the prescriptions given in, e.g., Bellini et al. (2017a) and Nardiello et al. (2018). We measured bright, isolated stars on the _drc exposures using aperture photometry with aperture of 5 pixels, and corrected for the finite aperture. We then computed the $2.5 \sigma$-clipped median value of the magnitude difference between our photometry and the aperture-corrected _drc-based magnitudes. Finally, we calibrated our photometry by adding to our instrumental magnitudes this median difference and the Vega-mag zero-point given in the STScI website. ${ }^{9}$

PMs were computed by combining the multi-epoch $H S T$ data as in Bellini et al. (2014), to which we refer for the detailed description of the method. We also corrected for spatially variable, high- and low-frequency systematic effects following the descriptions of Bellini et al. (2014, 2018) and Libralato et al. (2018). An overview of the final PM catalog of NGC 6352 is presented in Figure 1. The median 1D PM error of stars at the level of the MS turn-off $\left(18<m_{\mathrm{F} 606 \mathrm{~W}}<19\right)$ is

\footnotetext{
8 http://www.stsci.edu/ jayander/STDPSFs /

9 http://www.stsci.edu/hst/acs/analysis/zeropoints for ACS/WFC and http://www.stsci.edu/hst/wfc3/phot_zp_lbn for WFC3/UVIS.
}

of $\sim 32 \mu \mathrm{as} \mathrm{yr}^{-1}$. For comparison, stars in this field at the same magnitude level have a median 1D PM error of $\sim 360 \mu \mathrm{as} \mathrm{yr}^{-1}$ in the Gaia DR2. Figure 2 shows the PM along $\alpha \cos \delta$ and $\delta$ directions as a function of the $m_{\mathrm{F} 606 \mathrm{~W}}$ magnitude, $\left(m_{\mathrm{F} 606 \mathrm{~W}}-m_{\mathrm{F} 814 \mathrm{~W}}\right)$ color, $X$ and $Y$ master-frame positions. No clear systematic trends at levels comparable to the random errors for the best-measured stars arise from these plots.

Only objects measured in all the available filters of GO10775 and GO-13297 were considered in the analysis, thus the field of view (FOV) at disposal is that covered by the GO13297 WFC3/UVIS data. Stars are considered to be well measured if (i) their quality of PSF ( $Q F I T)$ parameter is larger than the 95 th percentile at any given magnitude, ${ }^{10}$ (ii) their magnitude rms is lower than the 95th percentile at any given magnitude, $^{11}$ (iii) they are measured in at least $40 \%$ of the images, (iv) their fraction of neighbor flux within the fitting radius before neighbor subtraction is less than $1,(\mathrm{v})$ their shape parameter RADXS (excess/deficiency of flux outside of the fitting radius with respect to the PSF prediction, see Bedin et al. 2008) is lower than the 85th percentile at any given magnitude, (vi) their flux is at least $3 \sigma$ above the local sky, (vii) their reduced $\chi^{2}$ of the PM fit is lower than 2 , and (viii) their rejection rate in the PM fit is lower than $30 \%$.

Finally, we also excluded stars with a PM error larger than half the local velocity dispersion $\sigma_{\mu}$ of the closest 100 cluster stars, and stars outside a radius of 75 arcsec from the cluster center (i.e., the four corners of the FOV) to avoid edge effects. These last two conditions are applied only in the kinematic analysis.

\section{Dissecting NGC 6352}

We identified the two populations of NGC 6352 in all evolutionary sequences by combining UV and optical CMDs and color-color diagrams, similarly to what is described in Milone et al. (2017) and Libralato et al. (2018).

We initially corrected for the differential reddening affecting our photometry following the prescription of, e.g., Milone et al. (2012) and Bellini et al. (2017b). NGC 6352 is in the direction of the Bulge and the extinction is high $(E(B-V)=0.22$, Harris 1996, 2010 edition). We assumed that cluster members are affected by the same amount of intra-cluster differential

\footnotetext{
${ }^{10}$ The closer the QFIT is to 1 , the better the PSF fit is. In addition to the 95th percentile cut, we consider as well measured all objects with a QFIT value higher than 0.99. We also discard all sources with QFIT lower than 0.6.

${ }^{11}$ Similarly as for the QFIT, we also keep all sources with a magnitude rms lower than $0.05 \mathrm{mag}$, and discard those with an rms higher than $0.4 \mathrm{mag}$.
} 

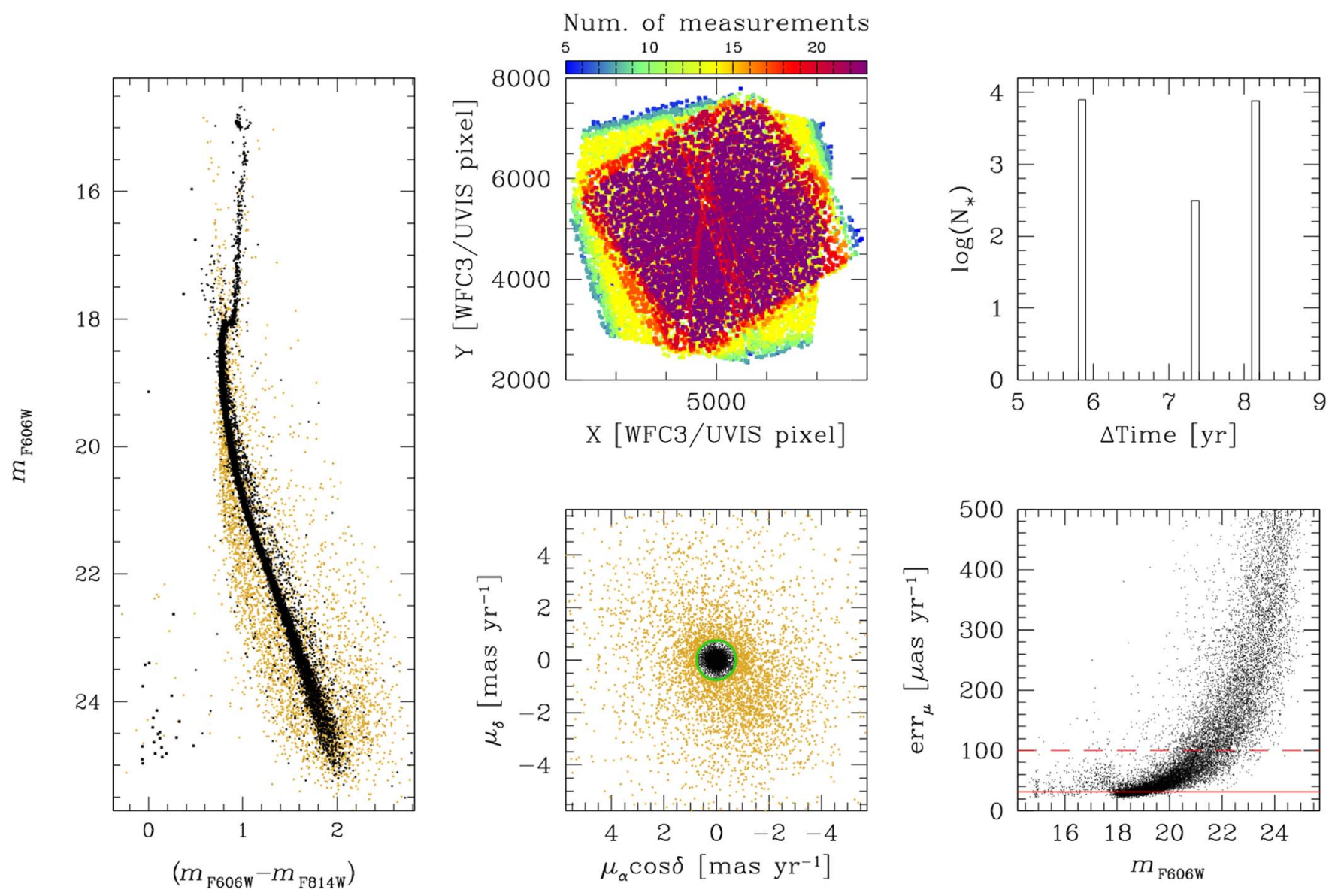

Figure 1. Overview of the PM catalog of NGC 6352. The $m_{\mathrm{F} 606 \mathrm{~W}}$ vs. $\left(m_{\mathrm{F} 606 \mathrm{~W}}-m_{\mathrm{F} 814 \mathrm{~W}}\right) \mathrm{CMD}$ (corrected for differential reddening; see Section 3$)$ is presented in the left panel. Cluster stars (defined as those with a PM within $0.75 \mathrm{mas} \mathrm{yr}^{-1}$ from the bulk motion of the cluster, i.e., $\sim 6$ times the central velocity dispersion) are shown in black, while field objects are plotted in yellow. In the bottom-middle panel, we show the vector-point diagram of the stars in the FOV. By construction, cluster stars

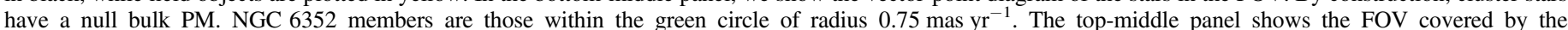
observations. The cluster is centered at $(5000,5000)$ WFC3/UVIS pixels. The center of the cluster is that defined by Goldsbury et al. (2010). Stars are color-coded as in the bar on top according to the number of measurements used in the PM fit. The logarithm of the number of sources as a function of the temporal baseline is shown

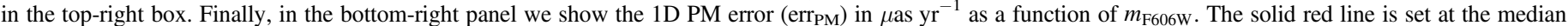
value of $\operatorname{err}_{\mathrm{PM}} \sim 32 \mu \mathrm{as} \mathrm{yr}^{-1}$ for the best-measured stars $\left(18<m_{\mathrm{F} 606 \mathrm{~W}}<19\right)$, while the dashed red line is set at 0.1 mas $^{-1}$ as reference.
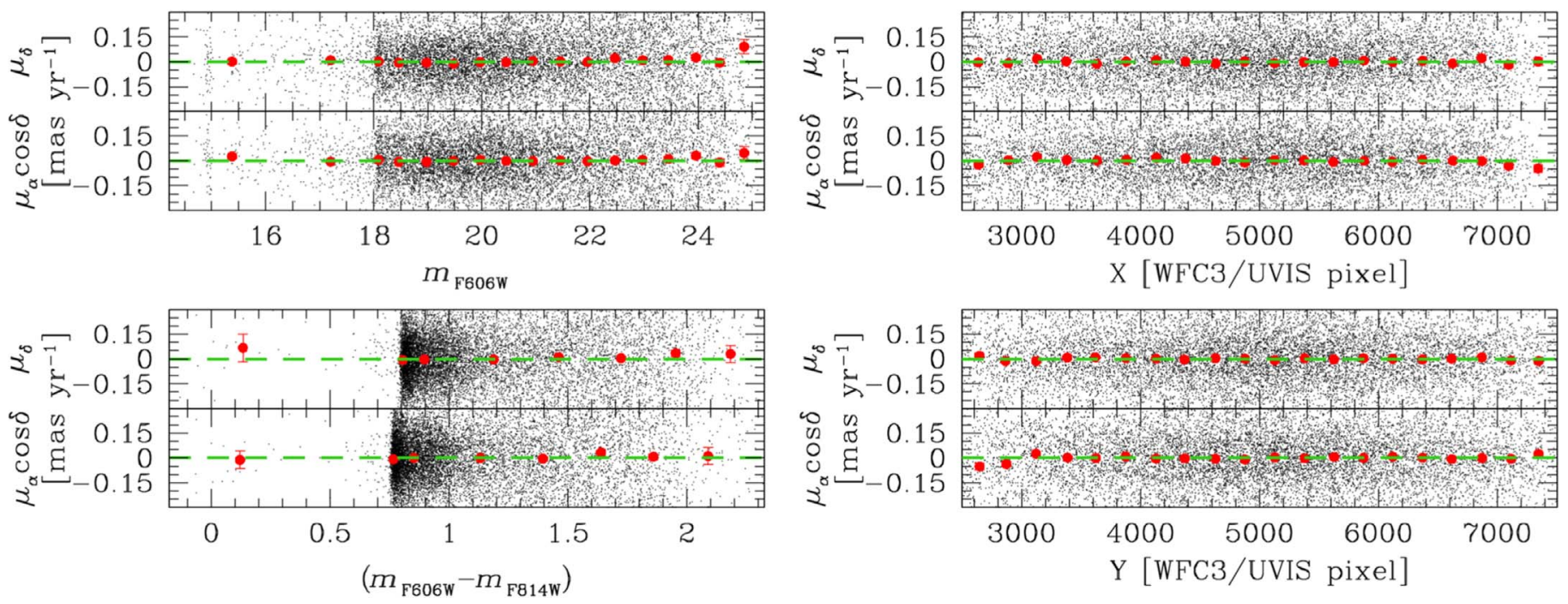

Figure 2. $\mu_{\alpha} \cos \delta$ and $\mu_{\delta}$ PMs as a function of the $m_{\mathrm{F} 606 \mathrm{~W}},\left(m_{\mathrm{F} 606 \mathrm{~W}}-m_{\mathrm{F} 814 \mathrm{~W}}\right), X$, and $Y$ master-frame positions. Only cluster stars with a PM lower than 0.75 mas yr ${ }^{-1}$ are shown. The red points (with error bars) are the $3.5 \sigma$-clipped median values of the PMs in different magnitude, color, or position bins. The green, dashed lines are set at $\mu_{\alpha} \cos \delta$ and $\mu_{\delta}$ equal to 0 . 

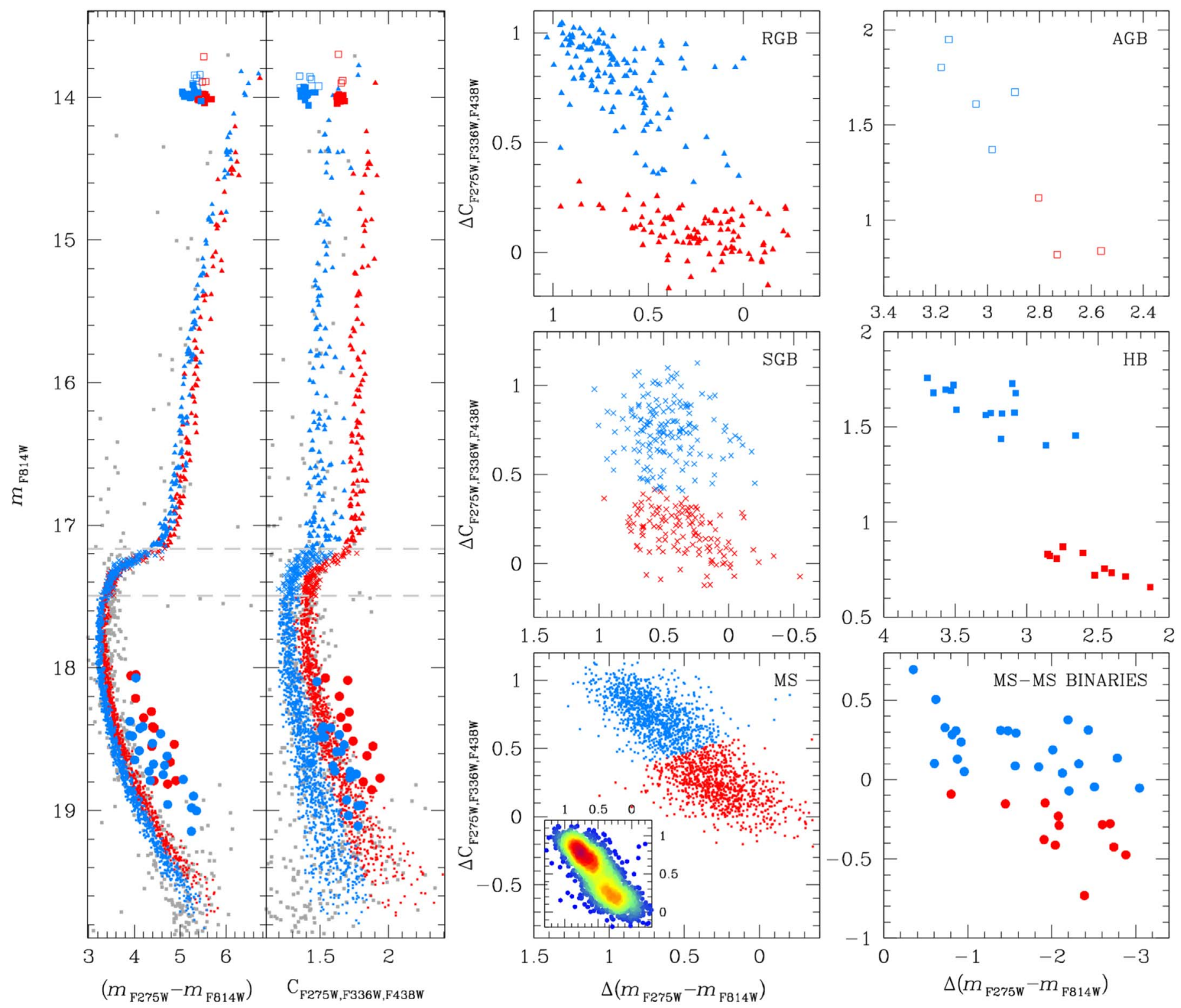

Figure 3. This figure illustrates the $\mathrm{mPOP}$ tagging performed to disentangle $\mathrm{POPa}$ (red points) and $\mathrm{POPb}$ (azure points). We rectified the $m_{\mathrm{F} 814 \mathrm{~W}} \mathrm{vs}$. $\left(m_{\mathrm{F} 275 \mathrm{~W}}-m_{\mathrm{F} 814 \mathrm{~W}}\right)$ and $m_{\mathrm{F} 814 \mathrm{~W}}$ vs. $\mathrm{C}_{\mathrm{F} 275 \mathrm{~W}, \mathrm{~F} 336 \mathrm{~W}, \mathrm{~F} 438 \mathrm{~W}} \mathrm{CMDs}$ (left panels). The gray, dashed horizontal lines in the left CMDs set the RGB, SGB, and MS separations. Gray dots are well-measured stars that are not selected as POPa,b stars or equal-mass binaries. We then computed the chromosome maps of each evolutionary sequence and separated $1 \mathrm{G}$ and $2 \mathrm{G}$ stars. For the MS stars, we made use of the Hess diagram in the inset of the MS chromosome map at this end. Equalmass MS binaries of POPa and $\mathrm{POPb}$ were defined as described in the text and in Figure 4.

reddening. For each star, we selected a sample of close-by cluster stars and measured their median shift along the reddening direction with respect to a fiducial line in the CMD. Since NGC 6352 hosts two distinct populations, we selected the most populous group $(\mathrm{POPb})$ to define the fiducial line. We tailored the correction for each CMD we present in this paper.

We first defined the RGB, SGB, and MS regions, and then separated POPa and $\mathrm{POPb}$ by means of the pseudo two-color diagrams "chromosome maps" (Milone et al. 2017). We drew by hand two fiducial lines along RGB, SGB, and MS in the $m_{\mathrm{F} 814 \mathrm{~W}}$ versus $\left(m_{\mathrm{F} 275 \mathrm{~W}}-m_{\mathrm{F} 814 \mathrm{~W}}\right)$ and $m_{\mathrm{F} 814 \mathrm{~W}}$ versus $C_{\mathrm{F} 275 \mathrm{~W}, \mathrm{~F} 336 \mathrm{~W}, \mathrm{~F} 438 \mathrm{~W}}=\left(m_{\mathrm{F} 275 \mathrm{~W}}-m_{\mathrm{F} 336 \mathrm{~W}}\right)-\left(m_{\mathrm{F} 336 \mathrm{~W}}-m_{\mathrm{F} 438 \mathrm{~W}}\right)$ planes, and rectified these sequences by defining $\Delta$ color $=$ $\left(\right.$ color-fiducial $\left._{\text {red }}\right) /\left(\right.$ fiducial $_{\text {blue }}-$ fiducial $\left._{\text {red }}\right)$, where "color" is either $\left(m_{\mathrm{F} 275 \mathrm{~W}}-m_{\mathrm{F} 814 \mathrm{~W}}\right)$ or $\mathrm{C}_{\mathrm{F} 275 \mathrm{~W}, \mathrm{~F} 336 \mathrm{~W}, \mathrm{~F} 438 \mathrm{~W}}$.
The chromosome maps of NGC 6352 are presented in Figure 3. The primordial $\mathrm{POPa}$ and the $2 \mathrm{G}$ stars of $\mathrm{POPb}$ are shown in red and azure, respectively. The two populations are quite obvious to separate in the RGB, SGB, AGB, and HB. The identification of the two populations in the MS is based on the Hess diagram of the chromosome map (inset in the MS panel of Figure 3).

We also separated equal-mass binaries that seem to belong to $\mathrm{POPa}$ and $\mathrm{POPb}$ as follows. First, we defined equal-mass MS binary objects $\sim 0.75 \mathrm{mag}$ brighter than the MS fiducial in the $m_{\mathrm{F} 814 \mathrm{~W}}$ versus $\left(m_{\mathrm{F} 606 \mathrm{~W}}-m_{\mathrm{F} 814 \mathrm{~W}}\right)$ CMD (left panel of Figure 4). Then, in the $m_{\mathrm{F} 814 \mathrm{~W}}$ versus $\mathrm{C}_{\mathrm{F} 275 \mathrm{~W}, \mathrm{~F} 336 \mathrm{~W}, \mathrm{~F} 438 \mathrm{~W}}$ $\mathrm{CMD}$, we tagged as POPa binaries the equal-mass binaries brighter than the reddest edge of the POPa MS. The remaining binaries belong to the $\mathrm{POPb}$ (right panel of Figure 4). Note that this classification for the equal-mass binaries does not consider 


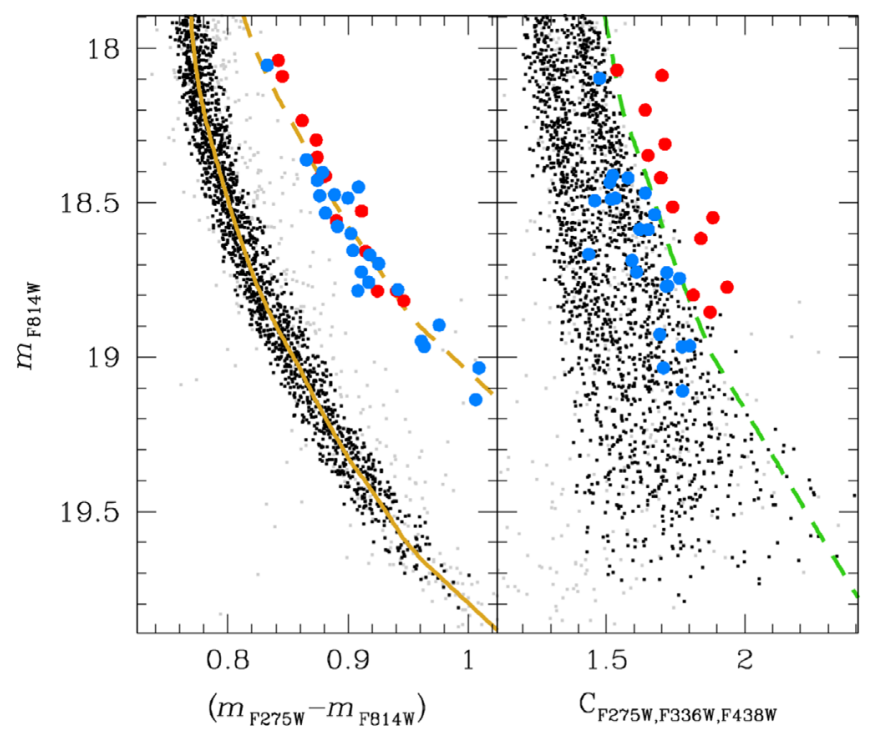

Figure 4. Overview of the mPOP tagging along the equal-mass MS binaries. In the $m_{\mathrm{F} 814 \mathrm{~W}}$ vs. $\left(m_{\mathrm{F} 606 \mathrm{~W}}-m_{\mathrm{F} 814 \mathrm{~W}}\right) \mathrm{CMD}$ (left panel), we initially selected as equal-mass MS binaries objects $0.75 \mathrm{mag}$ brighter than the MS fiducial line (yellow, solid line). The MS fiducial line shifted by $0.75 \mathrm{mag}$ is shown as a yellow, dashed line in the CMD as reference. Black points are stars belonging to either $\mathrm{POPa}$ or $\mathrm{POPb}$, while gray dots are all other objects. We then defined $\mathrm{POPa} / \mathrm{POPb}$ equal-mass MS binary stars brighter/fainter than the reddest edge (green, dashed line) of the POPa MS in the $m_{\mathrm{F} 814 \mathrm{~W}}$ vs. $\mathrm{C}_{\mathrm{F} 275 \mathrm{~W}, \mathrm{~F} 336 \mathrm{~W}, \mathrm{~F} 438 \mathrm{~W}}$ CMD (right panel).

the case of equal-mass mixed $1 \mathrm{G}+2 \mathrm{G}$ binaries, which are expected theoretically (e.g., Hong et al. 2016) and might have different properties from $1 \mathrm{G}+1 \mathrm{G}$ and $2 \mathrm{G}+2 \mathrm{G}$ systems.

\section{4. mPOPs Spatial Distribution}

Our HST observations of NGC 6352 cover an FOV out to $\sim 2 r_{\mathrm{c}}\left(r_{\mathrm{c}}=49.8\right.$ arcsec, Harris 1996, 2010 edition) from the cluster center. We analyzed the radial distributions of the populations along the RGB, SGB, and MS as follows. We chose as workbench the $m_{\mathrm{F} 814 \mathrm{~W}}$ versus $\mathrm{C}_{\mathrm{F} 275 \mathrm{~W}, \mathrm{~F} 336 \mathrm{~W}, \mathrm{~F} 438 \mathrm{~W}}$ CMD. We rectified the sequences by means of fiducial lines as described in Section 3. The fiducial lines were made by linearly interpolating the median color and magnitude in different $(0.5$, 0.1 , and $0.5 \mathrm{mag}$ for $\mathrm{RGB}, \mathrm{SGB}$, and $\mathrm{MS}$, respectively) magnitude bins.

We computed the histogram of the $\Delta \mathrm{C}_{\mathrm{F} 275 \mathrm{~W}, \mathrm{~F} 336 \mathrm{~W}, \mathrm{~F} 438 \mathrm{~W}}$ color adopting a bin width of $0.05 \mathrm{mag}$. This methodology is, however, sensitive to the bin width and the starting point of the histogram. To ensure a bias-free estimate of the fraction of $\mathrm{POPa}$ and POPb stars, we computed the histograms 10,000 times, each time adding a random noise to the points, and averaged them. The noise added to each star was randomly picked from a Gaussian distribution with $\sigma$ equal to the $\mathrm{C}_{\mathrm{F} 275 \mathrm{~W}, \mathrm{~F} 336 \mathrm{~W}, \mathrm{~F} 438 \mathrm{~W}}$ photometric error of the star. This way we removed the dependencies on the bin width (by blurring or sharpening the distributions) and on the starting point of the histogram (by shifting the stars in the rectified CMD).

The histograms of RGB, SGB, and MS stars present two distinct peaks. We fitted the final average histograms with a pair of Gaussian functions and estimated the fraction of stars belonging to $\mathrm{POPa}$ and $\mathrm{POPb}$ in a statistical fashion as described in Bellini et al. (2013).

Figures 5-7 present the radial distributions of the mPOPs along the RGB, SGB, and MS, respectively. The left panels show the $m_{\mathrm{F} 814 \mathrm{~W}}$ versus $\mathrm{C}_{\mathrm{F} 275 \mathrm{~W}, \mathrm{~F} 336 \mathrm{~W}, \mathrm{~F} 438 \mathrm{~W}} \mathrm{CMD}$ of the stars in the entire FOV. The rectified CMDs are displayed in the middle-left panels. The fiducial lines used to rectify the CMD are shown as azure and red lines. The average histogram and the dual Gaussian functions for all stars in the FOV are shown in the middle-right panels. The ratios $\mathrm{POPa}, \mathrm{b} /(\mathrm{POPa}+\mathrm{POPb})$ over the entire FOV are shown as red and azure solid horizontal lines, respectively, in the rightmost panels. The dashed horizontal lines indicate the $\pm 1 \sigma$ thresholds.

We repeated the same procedure shown in these three panels for stars in equally populated radial bins (two bins for the RGB, two for the SGB and five for the MS). The population ratio computed in each radial bin is shown with a filled circle in the rightmost panels of Figures 5-7. The number of bins in the RGB and SGB was chosen to have about 100 stars in each bin, while for the MS five bins are a good compromise between the need of a high statistics in each bin (about 360 stars per bin) and map possible radial features in the distributions. We also changed the number of radial bins for the MS and found consistent results within the error bars.

The ratios $\mathrm{POPa} /(\mathrm{POPa}+\mathrm{POPb})$ are listed in Table 2 . We find that the population ratio does not vary with the distance from the cluster center, and $\mathrm{POPb}$ is slightly more numerous than POPa. Our analysis is focused on the innermost regions of the cluster, which are the first parts where any initial radial gradient in the population ratio is erased by the effects of dynamical evolution, as shown in Vesperini et al. (2013). Our result is therefore consistent with the theoretical expectations. Considering that NGC 6352 has a relatively short half-mass relaxation time $\left(t_{\mathrm{hm}} \simeq 0.8 \mathrm{Gyr}\right.$, Harris 1996,2010 edition $)$, it is quite possible that the two populations are completely mixed over the entire cluster extension, but data covering a larger radial interval are necessary to further explore this issue.

Finally, it is worth noting that Milone et al. (2017) estimated the global fraction of $1 \mathrm{G} \mathrm{RGB}$ stars in NGC 6352. They found a ratio of $\mathrm{POPa} \mathrm{RGB}$ stars equal to $0.474 \pm 0.035$, in agreement with our independent estimate for the RGBa stars $(0.452 \pm 0.035)$.

$H B, A G B$, and equal-mass MS binaries - The number of stars in the AGB and $\mathrm{HB}$ is too small to perform an analysis of their radial distributions. However, the relative number of POPa stars is $\sim 38 \%$ and $40 \%$ of the total for AGB and HB, respectively, in agreement with the results for RGB, SGB, and MS. We also find that the relative number of POPa stars for the equal-mass $\mathrm{MS}$ binaries is $\sim 33 \%$.

In Figure 8, we present the cumulative radial distribution of equal-mass binaries of POPa and $\mathrm{POPb}$. The binary stars of the two populations are mixed in the cluster innermost regions. Recently, Hong et al. (2019) have shown that binary-star spatial mixing can be delayed by a number of dynamical processes affecting binaries (ionization, recoil, ejection). A more extended radial coverage than the one available in our HST data is required to test whether binaries are not mixed yet or NGC 6352 is sufficiently dynamically old to have reached complete spatial mixing also for its binary stars.

\section{Internal Kinematics of NGC 6352}

We analyzed the internal kinematics of the members of NGC 6352. In the following analysis, the velocity dispersions were obtained by correcting the observed scatter of the PMs for the uncertainties of the individual PMs as in van der Marel \&

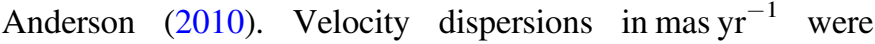



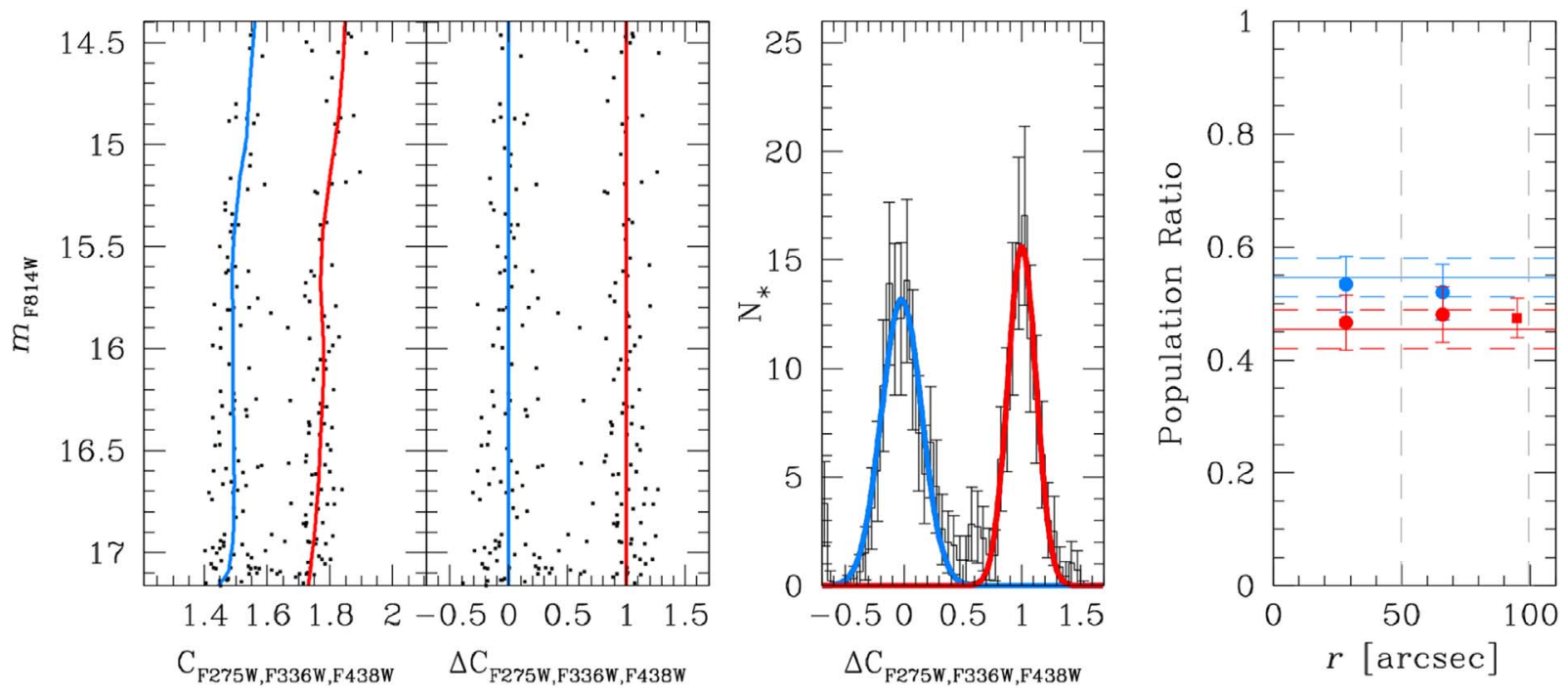

Figure 5. Left: $m_{\mathrm{F} 814 \mathrm{~W}}$ vs. $\mathrm{C}_{\mathrm{F} 275 \mathrm{~W}, \mathrm{~F} 336 \mathrm{~W}, \mathrm{~F} 438 \mathrm{~W}} \mathrm{CMD}$ of the RGB stars over the entire FOV. The azure and red lines represent the fiducial lines used to rectify the CMD in this magnitude range. Middle-left: $m_{\mathrm{F} 814 \mathrm{~W}}$ vs. $\Delta \mathrm{C}_{\mathrm{F} 275 \mathrm{~W}, \mathrm{~F} 336 \mathrm{~W}, \mathrm{~F} 438 \mathrm{~W}} \mathrm{CMD}$. Middle right: dual Gaussian fit of the $\Delta \mathrm{C}_{\mathrm{F} 275 \mathrm{~W}, \mathrm{~F} 336 \mathrm{~W}, \mathrm{~F} 438 \mathrm{~W}}$ distribution. The azure and red lines represent the individual Gaussian fit (the dual Gaussian fit is not shown because the two distributions are too separated). The histogram is the average of 10,000 histograms (see the text for details). Right: radial trends of the population ratio of RGBa (red filled circles) and RGBb (azure filled circles). The solid horizontal lines represent the value of the ratios obtained by considering all stars in the FOV. The dashed lines are set at $\pm 1 \sigma$ with respect to the solid lines. The two gray dashed vertical lines are set at $r_{\mathrm{c}}$ and $2 r_{\mathrm{c}}$. Finally, the red square (with error bars) marks the POPa ratio computed by Milone et al. (2017) for the same cluster.
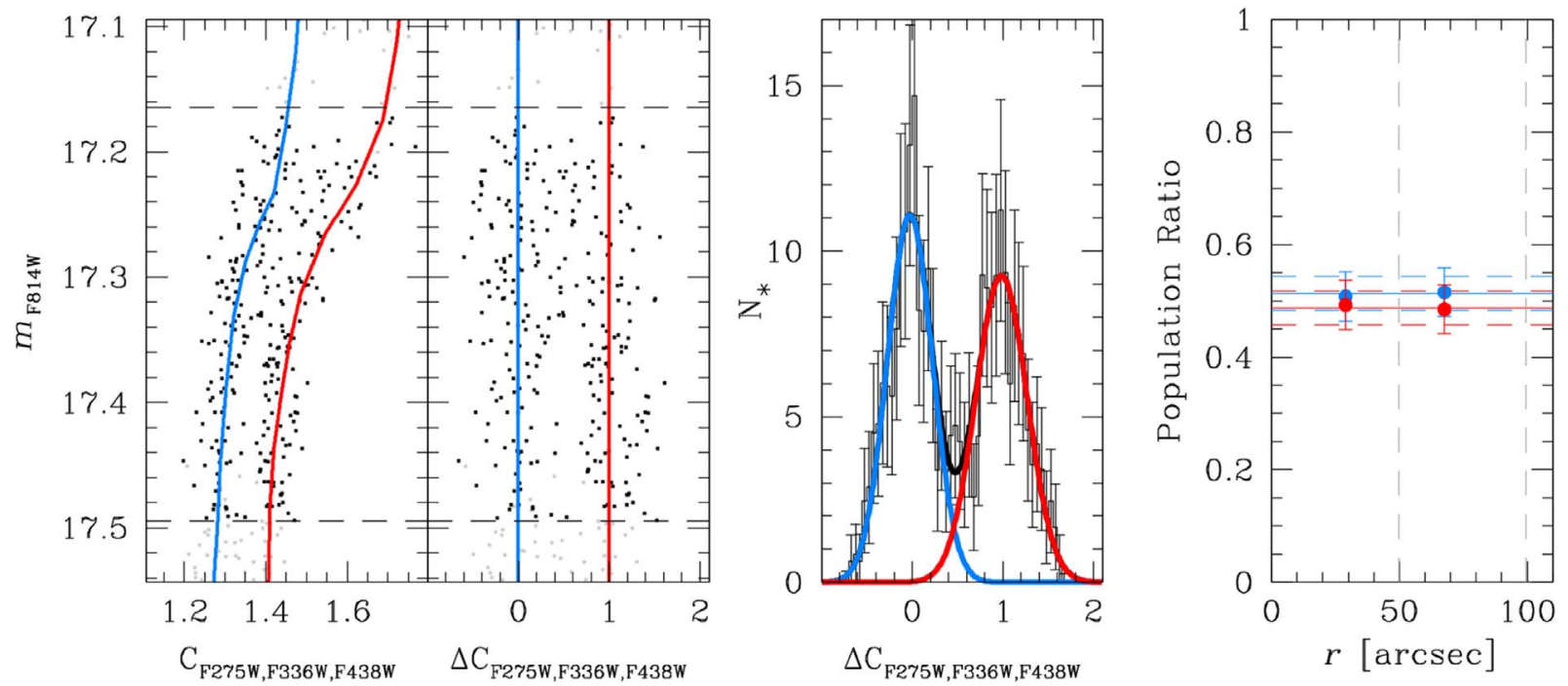

Figure 6. Similar to Figure 5, but for SGB stars. Stars not included in the SGB analysis (i.e., that are outside the magnitude interval defined by the two gray dashed horizontal lines in the left and middle-left CMDs) are shown with gray dots. The black line in the middle-right panel represents the dual Gaussian fit.

converted into $\mathrm{km} \mathrm{s}^{-1}$ by assuming a cluster distance of $5.6 \mathrm{kpc}$ (Harris 1996, 2010 edition).

\section{1. mPOP Kinematics}

We analyzed the combined $\left(\sigma_{\mu}\right)$, radial $\left(\sigma_{\mathrm{Rad}}\right)$, and tangential $\left(\sigma_{\text {Tan }}\right)$ velocity dispersions as a function of distance from the cluster center for each mPOP separately and in equally populated radial bins. Figure 9 presents the velocity dispersion (left) and anisotropy radial profiles (right) for the mPOPs along the RGB (top), SGB (middle), and MS (bottom), respectively. The value of each point is computed using 32 (43) stars for RGBa (RGBb), 55 (65) stars for SGBa (SGBb), and 145 (150) stars for $\mathrm{MSa}(\mathrm{MSb})$, respectively. As a reference, we also draw the average velocity dispersion and anisotropy of the entire sample (horizontal lines) and the corresponding $\pm 1 \sigma$ uncertainties (shaded regions).

Figure 9 shows that POPa (1G) and POPb (2G) have the same kinematics and are kinematically isotropic within $\sim 2 \sigma$. Considering that the data probe the cluster's innermost regions within $\sim 1.5 r_{\mathrm{c}}$, or $\sim 0.6 r_{\mathrm{h}}\left(r_{\mathrm{c}}=49.8\right.$ and $r_{\mathrm{h}}=123.6$ arcsec, Harris 1996, 2010 edition), this result is expected. The innermost regions are the first to relax and no significant anisotropy is, in general, expected close to the cluster's center (see, e.g., Tiongco et al. 2016). For example, Richer et al. (2013) and Bellini et al. (2015) found the presence of some velocity anisotropy in the $2 \mathrm{G}$ stars at distances larger than $1.5-2 r_{\mathrm{h}}$. A study of the kinematics in the cluster's outermost 

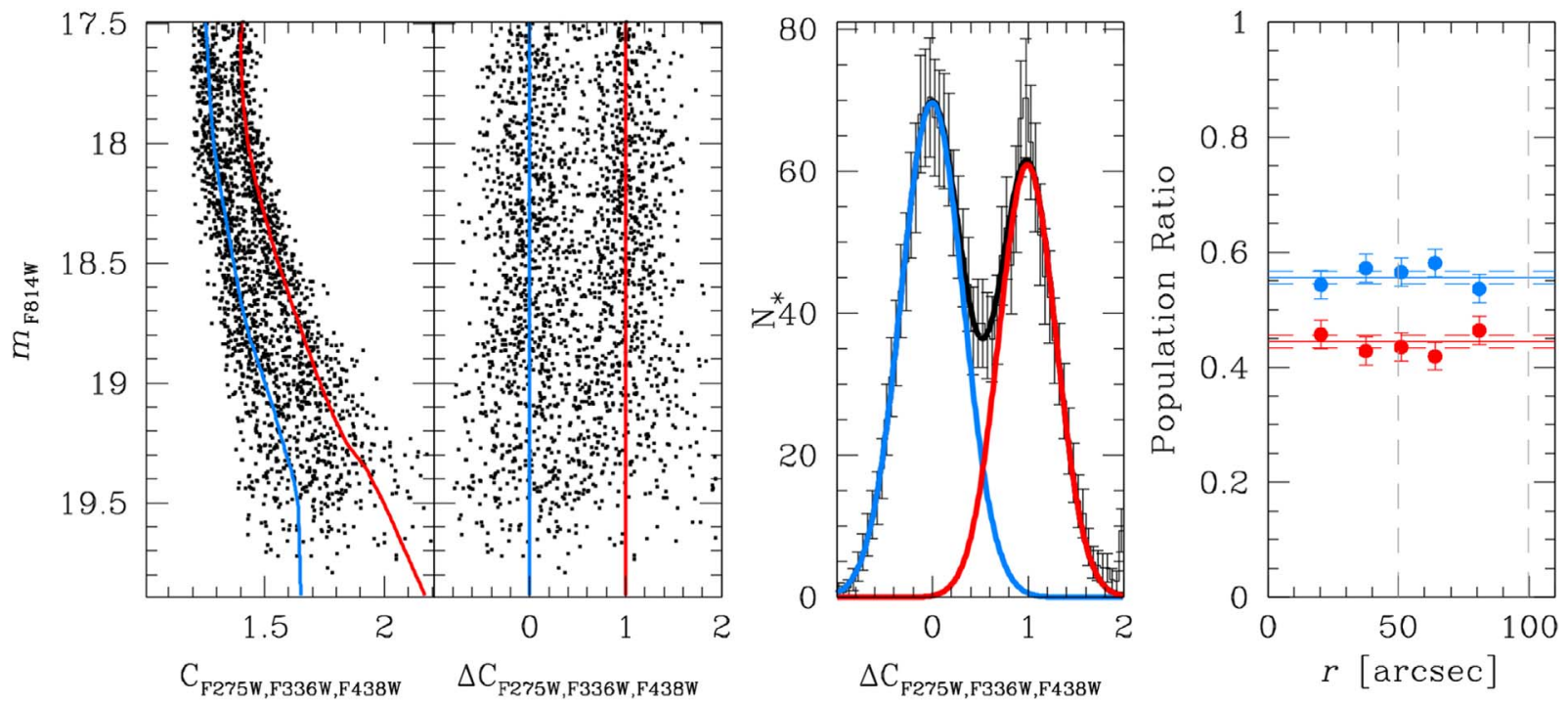

Figure 7. Similar to Figures 5 and 6, but for MS stars.

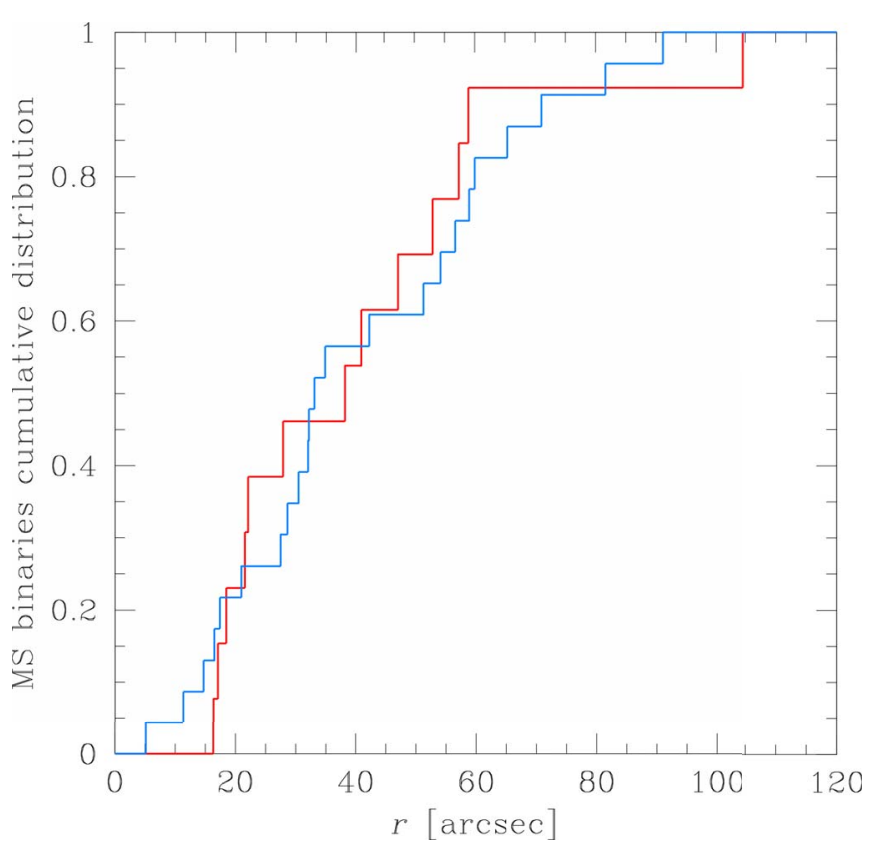

Figure 8. Cumulative distributions of equal-mass $1 \mathrm{G}$ (red) and $2 \mathrm{G}$ (azure) MS binaries.

regions would be necessary to further explore the possible presence of anisotropy in the velocity distribution due to internal dynamical processes and the cluster's interaction with the external tidal field (see, e.g., Tiongco et al. 2016).

$H B$ and equal-mass $M S$ binaries-We analyzed the internal kinematics of HB stars as a whole. Figure 10 shows $\sigma_{\mu}$ and the anisotropy for the HB stars (red and azure points for POPa and $\mathrm{POPb}$, respectively) as a function of radius. Again, the two populations hosted along the HB of NGC 6352 are isotropic and share the same kinematics within the errors.

We also computed the velocity dispersions of the mPOPs along the MS-binary sequence. Due to the low-number statistics, we made only one radial bin containing all stars in the FOV. The results are presented in Figure 11. The azure and red points show the velocity dispersion of MS binaries. MS
Table 2

Results of the Radial-distribution Analysis for NGC 6352

\begin{tabular}{lccc}
\hline \hline $\begin{array}{l}r_{\min } \\
(\operatorname{arcsec})\end{array}$ & $\begin{array}{c}r_{\max } \\
(\operatorname{arcsec})\end{array}$ & $\begin{array}{c}\bar{r} \\
(\operatorname{arcsec})\end{array}$ & $\mathrm{POPa} /(\mathrm{POPa}+\mathrm{POPb})$ \\
\hline 0.0 & 93.80 & 47.14 & $0.452 \pm 0.035$ \\
0.0 & 46.09 & 28.31 & $0.475 \pm 0.050$ \\
46.09 & 93.80 & 65.97 & $0.485 \pm 0.050$ \\
\hline & & $\mathrm{SGB}$ & \\
\hline 0.0 & 101.66 & 48.37 & $0.487 \pm 0.030$ \\
0.0 & 47.87 & 28.95 & $0.492 \pm 0.044$ \\
47.87 & 101.66 & 67.65 & $0.485 \pm 0.044$ \\
\hline & & $\mathrm{MS}$ & \\
\hline 0.0 & 103.29 & 50.77 & $0.445 \pm 0.011$ \\
0.0 & 30.34 & 20.34 & $0.457 \pm 0.025$ \\
30.34 & 44.11 & 37.49 & $0.428 \pm 0.024$ \\
44.11 & 57.89 & 51.04 & $0.435 \pm 0.025$ \\
57.89 & 70.55 & 63.98 & $0.419 \pm 0.024$ \\
70.55 & 103.29 & 80.93 & $0.464 \pm 0.025$ \\
\hline
\end{tabular}

binaries of $\mathrm{POPa}$ and $\mathrm{POPb}$ share the same kinematics and are isotropic.

We were not able to analyze AGB stars because of the very few stars at our disposal.

\section{2. $m P O P$ Differential Rotation}

We investigated the possible presence of differential rotation. We cannot directly measure any signature of the cluster's rotation in the plane of the sky because the systemic rotation signal is absorbed by the six-parameter linear transformations. However, there are different methods to infer the presence of rotation that rely on the skewness of the PMs in the tangential direction (see Heyl et al. 2017; Bellini et al. 2018; Libralato et al. 2018). Figure 12 shows the PMs of NGC 6352 in the $\mu_{\text {Tan }}$ versus $\mu_{\text {Rad }}$ plane.

We measured the amount of skew in the $\mu_{\text {Tan }}$ with (1) the value $G_{1}$ and the significance test $Z_{G 1}$ (Cramer 1997), and 

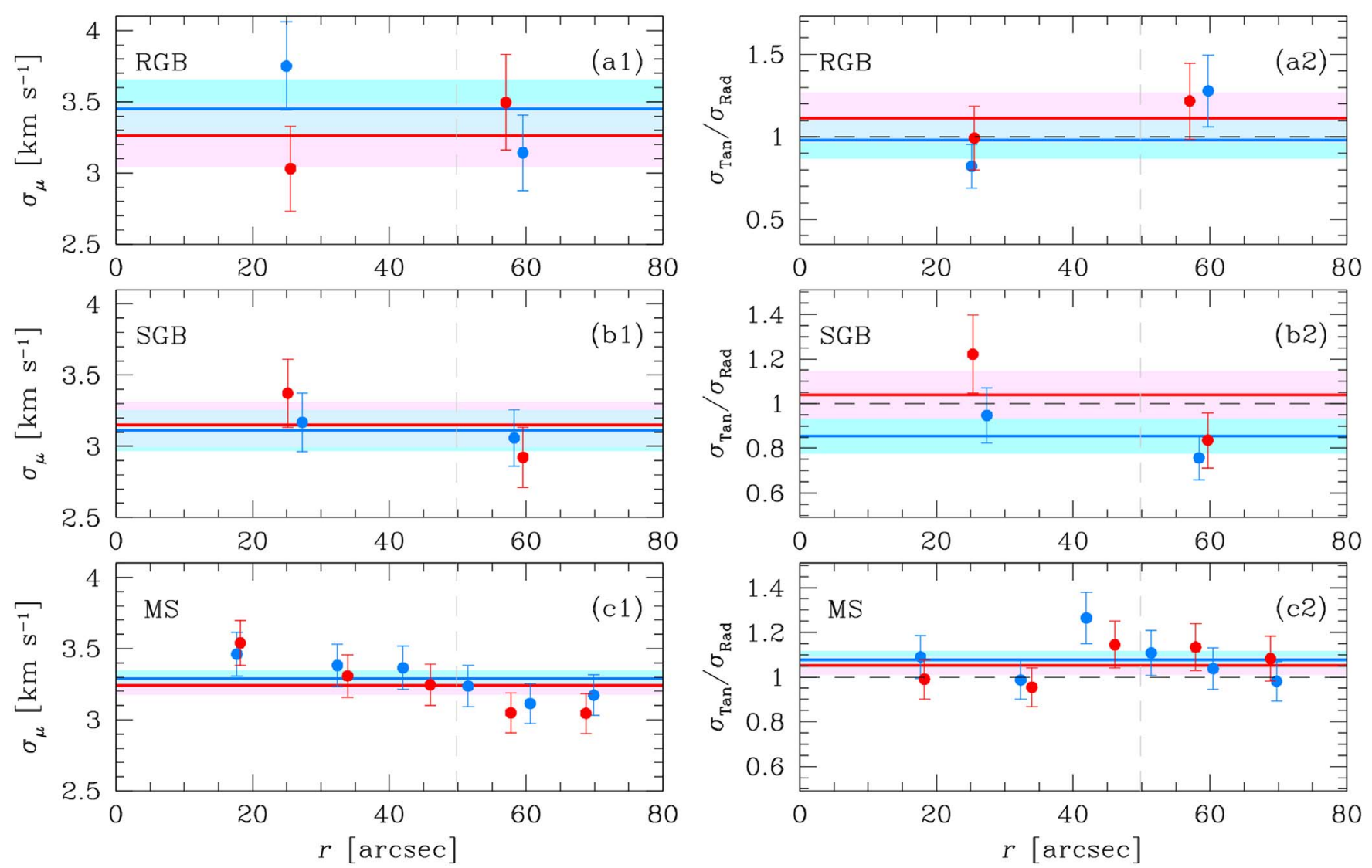

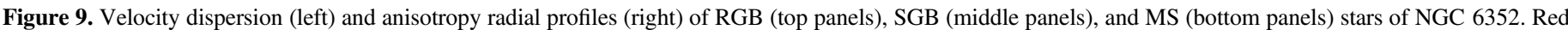

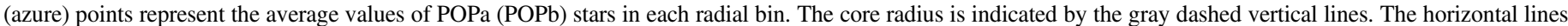

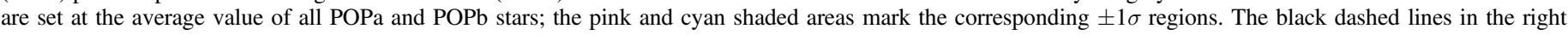
panels are set at 1 for reference.
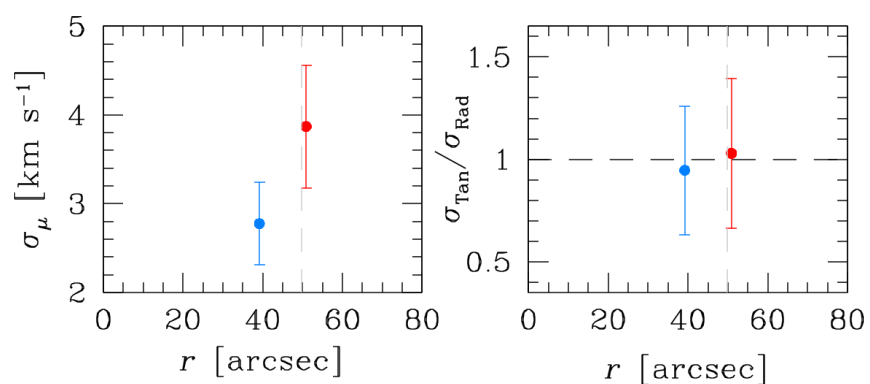

Figure 10. Combined velocity dispersion (left panel) and anisotropy (right panel) as a function of radius for the two populations in the HB of NGC 6352. Each bin is made up of $10 \mathrm{HB}$ stars.
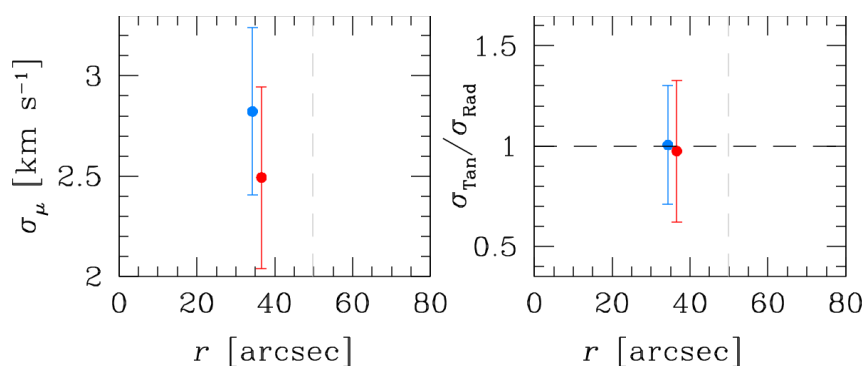

Figure 11. $\sigma_{\mu}$ as a function of distance from the cluster center for equal-mass MS binaries is shown in the left panel. Each bin represents the average value of all binaries (11 and 16 for POPa and $\mathrm{POPb}$, respectively). The right panel shows the tangential-to-radial anisotropy profile for the same stars.

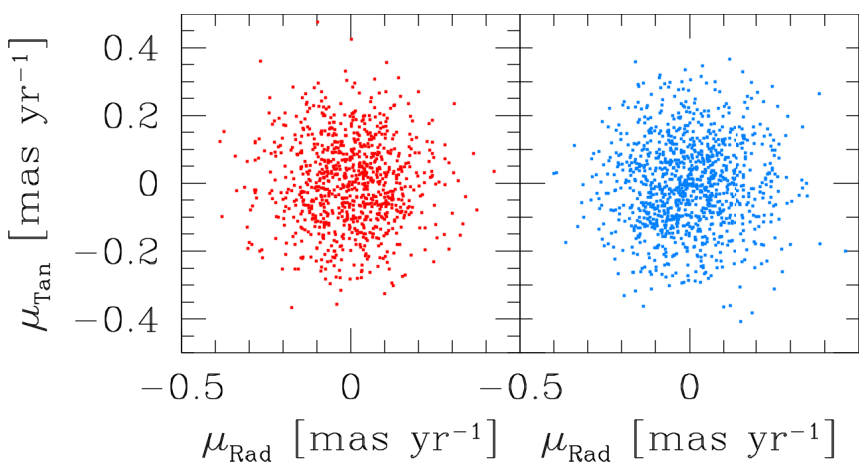

Figure 12. POPa (left panel) and POPb (right panel) PMs in the $\mu_{\text {Tan }}$ vs. $\mu_{\mathrm{Rad}}$ plane.

(2) the third-order Gauss-Hermite moment $h_{3}$ (van der Marel \& Franx 1993). We find

$$
\begin{aligned}
& \text { POPa: }\left\{\begin{array}{l}
G_{1}=0.09, Z_{G_{1}}=1.05 \\
h_{3}=0.021 \pm 0.026
\end{array},\right. \\
& \text { POPb: }\left\{\begin{array}{l}
G_{1}=0.09, Z_{G_{1}}=1.27 \\
h_{3}=0.021 \pm 0.024
\end{array} .\right.
\end{aligned}
$$

These values are consistent with an absence of differential rotation in NGC 6352. 

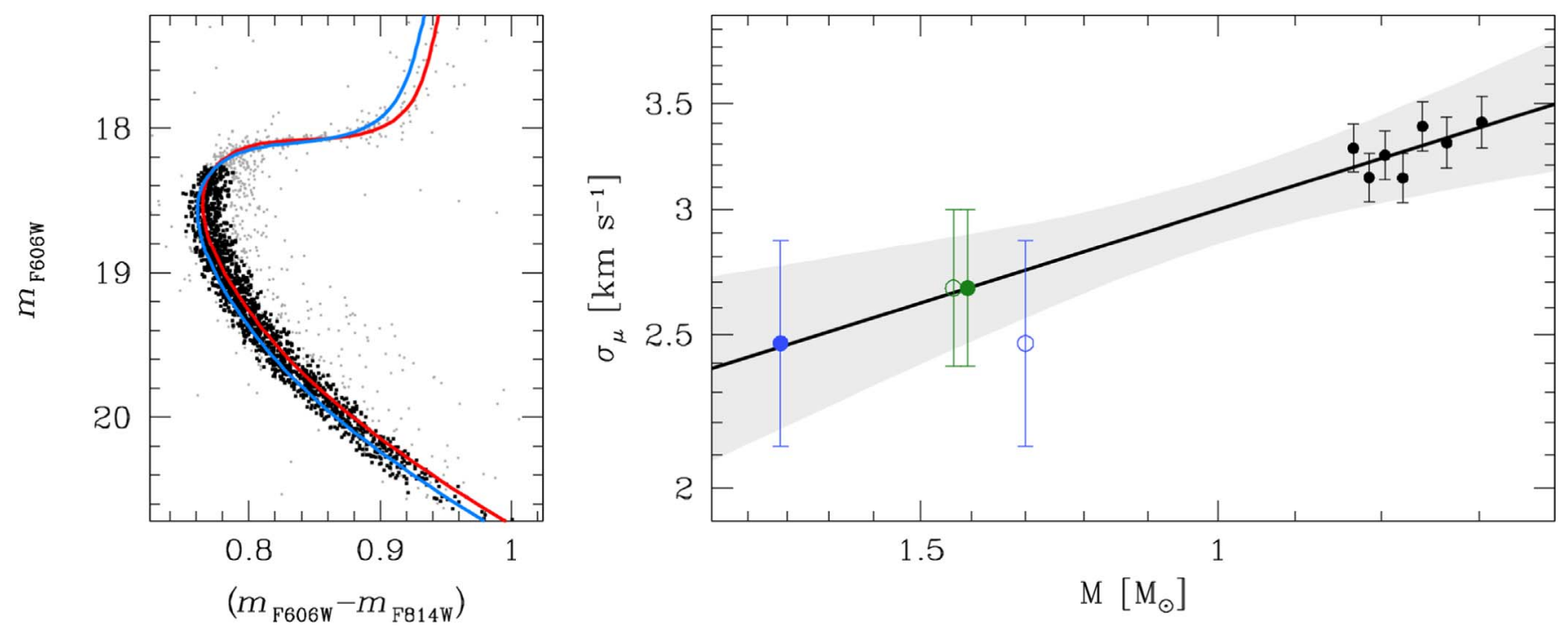

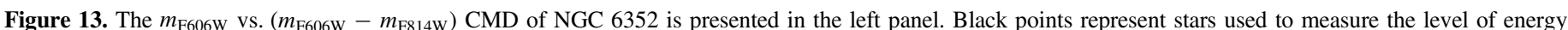

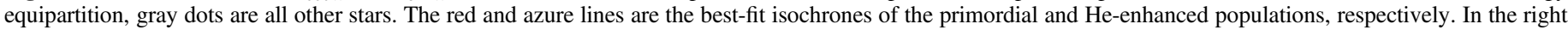

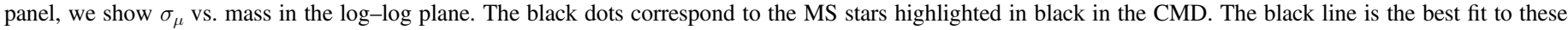

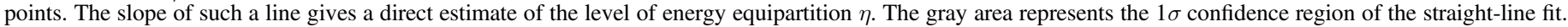

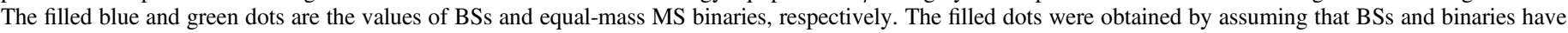

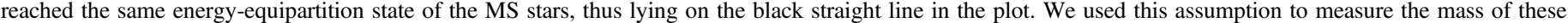

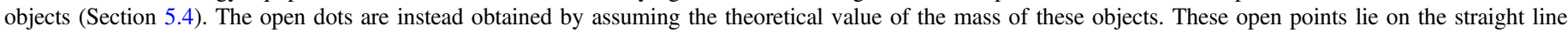
within $1 \sigma$, meaning that BS and equal-mass MS binaries have the same level of energy equipartition of lower-mass members of NGC 6352.

Recently, Bianchini et al. (2018) estimated the amount of rotation in GCs by means of the Gaia DR2 PMs. The authors measured for NGC $6352 \mu_{\operatorname{Tan}}\left(r<3 r_{\mathrm{h}}\right)=0.001_{-0.013}^{+0.014} \mathrm{mas} \mathrm{yr}^{-1}$ and a $1 \sigma$ upper limit of $V / \sigma=0.09$ ( $V$ is the value of the rotation peak, $\sigma$ is the central velocity dispersion): values consistent with no rotation in the plane of the sky. Furthermore, they found a correlation between $V / \sigma$ and the relaxation time.

As GCs advance in their evolution, they gradually lose their initial rotation (see, e.g., Ernst et al. 2007; Tiongco et al. 2017, and references therein). The absence of rotation in NGC 6352 could be another indication of its advanced dynamical age.

\subsection{Level of Energy Equipartition}

GCs evolve toward a state of increasing energy equipartition $\left(\sigma_{\mu} \propto m^{-\eta}\right.$ with $m$ the stellar mass and $\eta=0.5$ for a complete energy equipartition), but they are not expected to reach a complete equipartition (Trenti \& van der Marel 2013; Bianchini et al. 2016; Webb \& Vesperini 2017).

We divided the MS into seven equally populated magnitude bins, and computed $\sigma_{\mu}$ and median magnitude of the $\sim 230$ stars in each bin. We then transformed these magnitudes into stellar masses by using a set of Dartmouth isochrones (Dotter et al. 2008).

NGC 6352 has an age of 13.0 Gyr (e.g., Wagner-Kaiser et al. 2017), $[\mathrm{Fe} / \mathrm{H}]=-0.67$ (Carretta \& Gratton 1997), $[\alpha / \mathrm{Fe}]=0.4$, $E(B-V)=0.22$, and it is at a distance of $5.6 \mathrm{kpc}$ (Harris 1996, 2010 edition). Nardiello et al. (2015) analyzed the He content and relative age of the two populations hosted in NGC 6352, finding $\Delta Y=0.029 \pm 0.006$ and $\Delta$ Age $=10 \pm 120 \mathrm{Myr}$.

The best-fit isochrones are derived using a technique similar to that described in Nardiello et al. (2015) to estimate the He difference between $\mathrm{POPa}$ and $\mathrm{POPb}$ in NGC 6352. We compared the colors of the observed fiducial lines of the two populations in the $m_{\mathrm{F} 606 \mathrm{~W}}$ versus $\left(m_{\mathrm{F} 606 \mathrm{~W}}-m_{\mathrm{F} 814 \mathrm{~W}}\right) \mathrm{CMD}$ with the colors of the fiducial lines of synthetic CMDs built from a set of isochrones with $[\mathrm{Fe} / \mathrm{H}]=-0.67$ and $[\alpha / \mathrm{Fe}]=0.4$. We treated both mPOPs at the same time, using the same isochrones except for the He content $(Y=0.256$ for $\mathrm{POPa}$ and $Y=0.285$ for $\mathrm{POPb}$ ). We let distance, reddening, and age vary, and defined the best-fit isochrones as those that provided the lowest $\chi^{2}$. We find

$$
\left\{\begin{aligned}
E(B-V) & =0.25 \\
\text { Distance } & =5.3 \mathrm{kpc} \\
\text { Age } & =13.0 \mathrm{Gyr} .
\end{aligned}\right.
$$

The best isochrones for POPa and $\mathrm{POPb}$ are shown in the left panel of Figure 13 as red and azure lines, respectively. The MS of NGC 6352 is made up by $45 \%$ of POPa stars and $55 \%$ of $\mathrm{POPb}$ stars. Therefore, we weighted the median mass in each magnitude bin by these ratios.

Finally, we fitted the $\sigma_{\mu}$ versus mass values in a log-log plane with a weighted least-square straight line and find

$$
\eta=0.334 \pm 0.182
$$

The result is presented in the right panel of Figure 13. The black points picture the velocity dispersions of the seven MS bins; the black line is the best fit to these points (we postpone the explanation of the azure and green points to the next section). We computed the value of $\eta$ by using isochrones with slightly different ages, $[\mathrm{Fe} / \mathrm{H}]$, and by neglecting the presence of the mPOPs, and obtained consistent results within the errors.

This value of $\eta$ provides further evidence that NGC 6352 is in an advanced stage of its dynamical evolution, and it is in general consistent with the theoretical predictions of Trenti \& van der Marel (2013) and Webb \& Vesperini (2017), although our uncertainty on the value of $\eta$, mainly due to the low statistics and the small mass range considered, is large. 

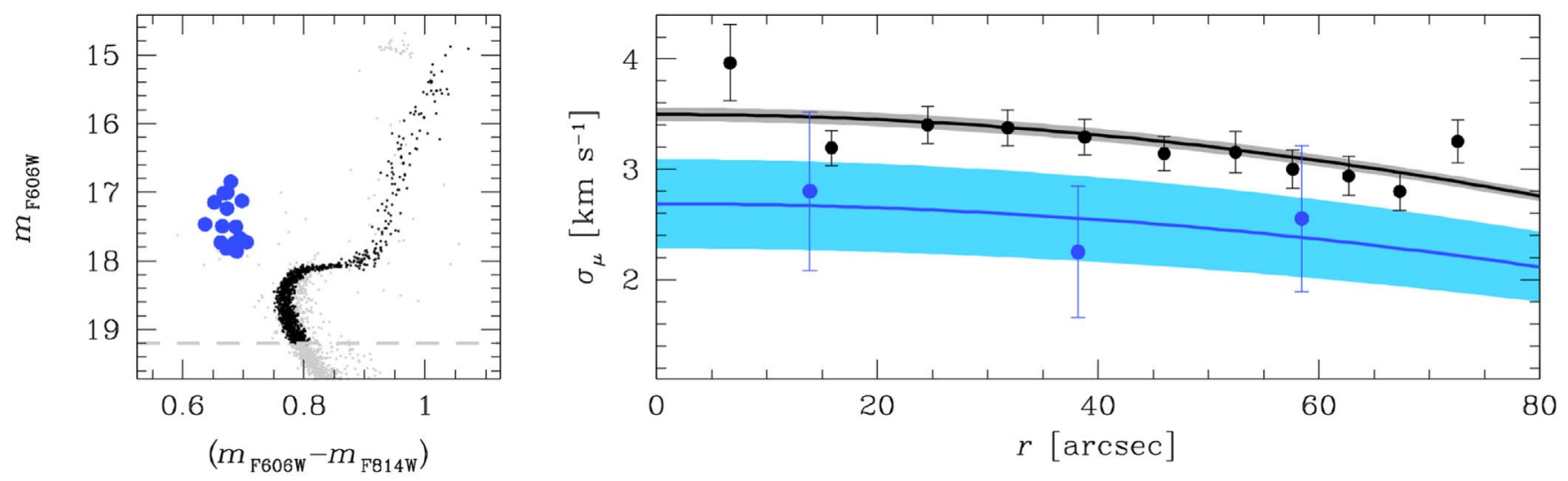

Figure 14. Highlight of the procedure adopted to measure the mass of the BSs. We compared the combined velocity dispersion of the BSs (blue dots in the $m_{\mathrm{F} 606 \mathrm{~W}}$ vs. $\left(m_{\mathrm{F} 606 \mathrm{~W}}-m_{\mathrm{F} 814 \mathrm{~W}}\right) \mathrm{CMD}$ in the left panel) with that of all stars brighter than 0.5 mag below the MS turn-off (black dots brighter than the gray, dashed line in the CMD; other objects are shown as gray dots). The right panel shows the velocity dispersion of BSs (blue dots) and of the reference population (black dots). It is clear that massive objects move slower than lighter stars because of the energy equipartition. We fit the velocity dispersion radial profile of the reference population (black line), and then found the value $\alpha$ that provides the best fit of the same profile on the BS kinematics (blue line). The gray and pale-blue regions set the $1 \sigma$ errors of the fitted profiles for reference stars and BSs, respectively.
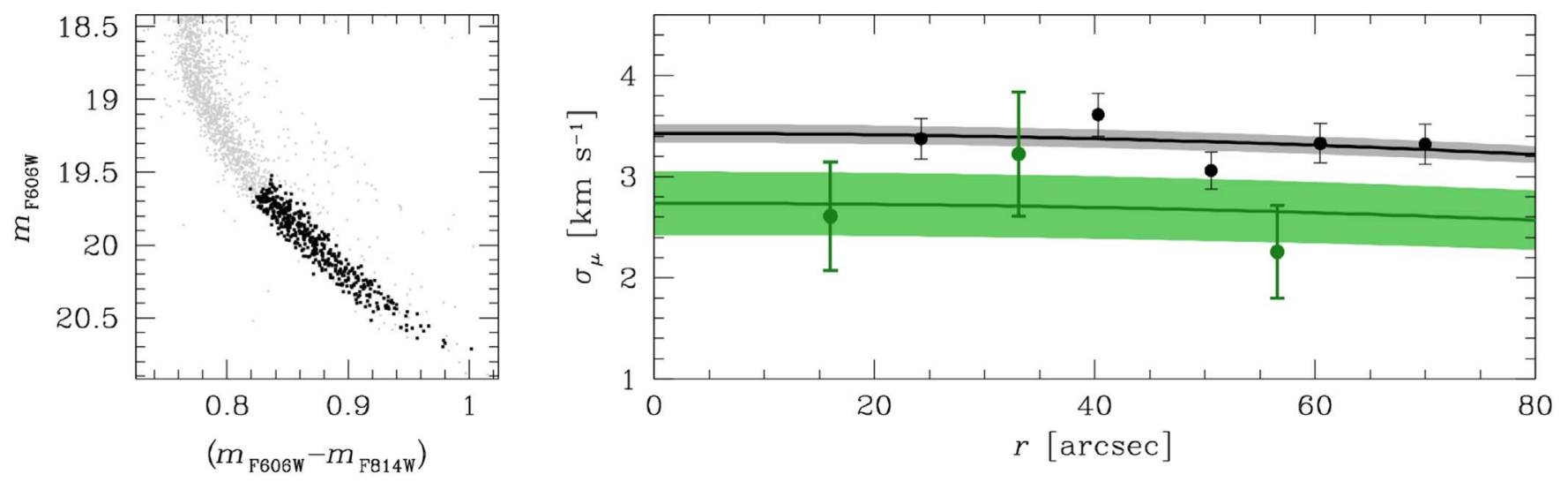

Figure 15. Similar to Figure 14, but for the equal-mass MS binaries.

\subsection{The Mass of Blue Stragglers and MS Binaries}

Blue stragglers (BSs) and MS-binary systems are more massive than a typical (single) star in a GC. If the GC has some degree of energy equipartition, we expect these massive objects to have a lower velocity dispersion than the other stars. Baldwin et al. (2016) computed the mass of the BSs in several GCs by assuming that the velocity dispersions and masses of these stars scale as

$$
\alpha=\frac{\sigma_{\mathrm{BSS}}}{\sigma_{\mathrm{MSTO}}}=\left(\frac{M_{\mathrm{BSS}}}{M_{\mathrm{MSTO}}}\right)^{-\eta} .
$$

We calculated the mass of the BSs in NGC 6352 in a similar fashion, and extended the same methodology to the equal-mass MS binaries.

For the BSs (blue dots in the left panel of Figure 14), we computed the combined velocity dispersion of these stars into three equally populated radial bins of five stars each. We chose as a reference population all RGB, SGB, and MS stars brighter than 0.5 mag below the MS turn-off (black points in the CMD in Figure 14), and computed the velocity dispersions of these reference objects in 11 radial bins. ${ }^{12}$ The velocity dispersion

\footnotetext{
12 One bin of 37 stars was defined by all stars within 10 arcsec from the center of the GC. Five equally populated bins of 118 stars each were defined from 10 arcsec to the core radius, and five bins of 81 stars each from the core radius to the limit of the analyzed FOV.
}

radial profiles of BSs and reference stars are shown in the right panel of Figure 14 in blue and black, respectively.

For each radial bin of the reference population, we drew 10,000 realizations of the average velocity dispersion by adding a random Gaussian noise with $\sigma$ equal to the error in the velocity dispersion. We then interpolated the median values of these distributions by means of a third-order polynomial function with a flat center. The best fit is shown in Figure 14. The gray region represents the $1 \sigma$ error of the fitted profiles.

We performed a similar computation for the BS, but this time we interpolated the velocity dispersion profile with the same third-order polynomial function of the reference population rescaled by a factor $\alpha$. The blue line in Figure 14 depicts the best fit for the BSs, the pale-blue region is the $1 \sigma$-error region.

We find $\alpha=0.77 \pm 0.12$. Given an MS turn-off mass of $0.83 M_{\odot}$ and assuming that the SGB and RGB stars have the same dynamical mass of those at the MS turn-off, we derive a mass for the BSs of $1.82 \pm 0.37 M_{\odot}$. Our estimate is slightly larger, although in agreement at the $1 \sigma$ level, than the typical mass of a BS (between 1.0 and $1.6 M_{\odot}$; see, e.g., Ferraro et al. 2018).

We also estimated the average mass of the equal-mass MS binary systems (green dots in Figure 15) as done for the BSs. This time we chose as reference the MS stars 0.75 mag fainter 


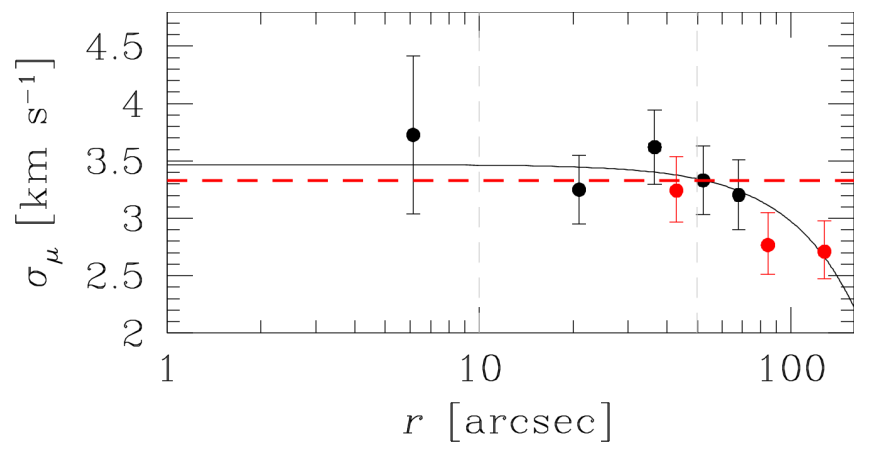

Figure 16. Combined velocity dispersion as a function of distance from the cluster center for RGB stars. Black points represent the kinematics of the RGB stars measured in this work, red points are those obtained by Baumgardt et al. (2019) with the Gaia DR2 PMs. The black line is the best fit to our data. The red, dashed line is set at the central value of the velocity dispersion computed by Baumgardt et al. (2019).

than the equal-mass binary region (black points in Figure 15). We find $\alpha=0.80 \pm 0.09$. The average mass of the MS stars (computed as described in Section 5.3) in the magnitude interval considered in the analysis is $\sim 0.72 M_{\odot}$, which gives us an average mass of the binary systems of $1.41 \pm 0.30 M_{\odot}$. This is consistent with a system made by two stars of $0.72 M_{\odot}$ each.

Returning back to Figure 13, its right panel shows the average velocity dispersion of all BSs and MS binaries as a function of mass. The filled dots refer to the mass computed in this section and, by construction, they are aligned with the best fit of the MS stars from which we computed the value of $\eta$. If we assume an average mass of the BSs and MS binaries of $1.3 M_{\odot}$ (the midrange value of typical BS masses from Ferraro et al. 2018) and $1.44 M_{\odot}$ (twice the average MS stars at the same color level of the binaries), respectively, we can have an independent check of the goodness of our estimate of $\eta$. The empty dots plotted by assuming these theoretical values of the mass for BSs and MS-binary systems seem to be in agreement with the prediction of the MS stars (black line), and confirm the level of energy equipartition we computed with this $H S T$ data set.

\subsection{Comparison with the Literature}

As an external check, we made a comparison with the work of Baumgardt et al. (2019). The authors fitted $N$-body models to ground-based radial velocities, HST-based mass functions, and the Gaia DR2 PMs, and derived structural parameters for 144 Galactic GCs. ${ }^{13}$

First, we compared our $H S T$-based velocity dispersion radial profiles with those obtained with the Gaia DR2. We limited our analysis to the RGB stars to be consistent with Baumgardt et al. (2019). We computed the average $\sigma_{\mu}$ of all stars within $10 \operatorname{arcsec}$ from the cluster center, and in four equally populated (35 stars per bin) radial bins in the remaining part of the FOV. The values of $\sigma_{\mu}$ computed in this work are shown in black in Figure 16. The red points are the velocity dispersions computed with the Gaia DR2 PMs by Baumgardt et al. (2019), rescaled to the cluster distance adopted in our work. The HST- and Gaia-based velocity dispersions are in excellent agreement with each other.

Baumgardt et al. (2019) provided an estimate of the central velocity dispersion $\sigma_{0}=3.5 \mathrm{~km} \mathrm{~s}^{-1}$, or $\sigma_{0}=3.33 \mathrm{~km} \mathrm{~s}^{-1}$ upon rescaling for the distance adopted in our work. We fitted

\footnotetext{
${ }^{13}$ https://people.smp.uq.edu.au/HolgerBaumgardt/globular/
}

our velocity dispersion radial profiles with a third-order polynomial (forced to be flat at the center) and extrapolated a value of $\sigma_{0}=3.47 \mathrm{~km} \mathrm{~s}^{-1}$, consistent with the value of Baumgardt et al. (2019).

Finally, Baumgardt et al. (2019) also computed the value of $\eta$ for stars in the mass range $0.5-0.8 M_{\odot}$. They find $\eta=0.36$, which is consistent with the value we derived from our HSTbased PMs in Section 5.3.

\section{Conclusions}

In this paper we presented a comprehensive characterization of the structural and kinematic properties of the mPOPs in the innermost regions of the Galactic GC NGC 6352.

We used data from the HST UV survey GO-13297 to identify $1 \mathrm{G}(\mathrm{POPa})$ and $2 \mathrm{G}(\mathrm{POPb})$ stars along the RGB, SGB, and MS, finding a similar fraction of POPa stars $(\sim 45 \%)$ in each evolutionary group. We also find no evidence of a significant variation in the fraction of POPa stars with distance from the cluster center. As shown in previous theoretical studies, the innermost regions are the first where any initial radial gradients is erased, and the lack of a radial variation in the fraction of stars belonging to the two populations is therefore consistent with the theoretical expectations.

We studied the cluster's internal kinematics by means of HST-based, high-precision PMs. There is no sign of internal rotation, anisotropy in the velocity distributions, and differences between the kinematic properties of $\mathrm{POPa}$ and $\mathrm{POPb}$ stars. We also measured the dependence of the velocity dispersion on the stellar mass, and provided a quantitative estimate of the level of energy equipartition reached in the cluster's inner regions.

NGC 6352 is probably in its advanced evolutionary stages, and any difference in the structural and kinematic properties of mPOPs might have been erased by dynamical processes over the entire cluster's extension. It will be essential to extend the analysis presented in this paper to the cluster' outer regions where some memories of the initial or dynamically induced differences might still be retained.

M.L. and A.B. acknowledge support from STScI grant GO13297. The authors thank the anonymous referee for the thoughtful suggestions that improved the quality of the paper. Based on observations with the NASA/ESA HST, obtained at the Space Telescope Science Institute, which is operated by AURA, Inc., under NASA contract NAS 5-26555. This work has made use of data from the European Space Agency (ESA) mission Gaia (https://www.cosmos.esa.int/gaia), processed by the Gaia Data Processing and Analysis Consortium (DPAC, https:// www.cosmos.esa.int/web/gaia/dpac/consortium). Funding for the DPAC has been provided by national institutions, in particular, the institutions participating in the Gaia Multilateral Agreement.

\section{ORCID iDs}

Mattia Libralato (1) https://orcid.org/0000-0001-9673-7397 Andrea Bellini (ib https://orcid.org/0000-0003-3858-637X Giampaolo Piotto (1) https://orcid.org/0000-0002-9937-6387 Domenico Nardiello (i) https://orcid.org/0000-00031149-3659

Roeland P. van der Marel (ib https://orcid.org/0000-00017827-7825 
Jay Anderson (1) https://orcid.org/0000-0003-2861-3995

Luigi R. Bedin (1) https://orcid.org/0000-0003-4080-6466

Enrico Vesperini (i) https://orcid.org/0000-0003-2742-6872

\section{References}

Anderson, J., \& Bedin, L. R. 2010, PASP, 122, 1035

Anderson, J., \& King, I. R. 2006, Instrument Science Report ACS 2006-01, http://www.stsci.edu/hst/acs/documents/isrs/isr0601.pdf

Anderson, J., \& van der Marel, R. P. 2010, ApJ, 710, 1032

Baldwin, A. T., Watkins, L. L., van der Marel, R. P., et al. 2016, ApJ, 827, 12

Baumgardt, H., Hilker, M., Sollima, A., \& Bellini, A. 2019, MNRAS, 482, 5138

Bedin, L. R., King, I. R., Anderson, J., et al. 2008, ApJ, 678, 1279

Bellini, A., Anderson, J., \& Bedin, L. R. 2011, PASP, 123, 622

Bellini, A., Anderson, J., Bedin, L. R., et al. 2017a, ApJ, 842, 6

Bellini, A., Anderson, J., van der Marel, R. P., et al. 2014, ApJ, 797, 115

Bellini, A., Anderson, J., van der Marel, R. P., et al. 2017b, ApJ, 842, 7

Bellini, A., \& Bedin, L. R. 2009, PASP, 121, 141

Bellini, A., Libralato, M., Bedin, L. R., et al. 2018, ApJ, 853, 86

Bellini, A., Piotto, G., Milone, A. P., et al. 2013, ApJ, 765, 32

Bellini, A., Vesperini, E., Piotto, G., et al. 2015, ApJL, 810, L13

Bianchini, P., van de Ven, G., Norris, M. A., Schinnerer, E., \& Varri, A. L. 2016, MNRAS, 458, 3644

Bianchini, P., van der Marel, R. P., del Pino, A., et al. 2018, MNRAS, 481, 2125

Carretta, E., \& Gratton, R. G. 1997, A\&AS, 121, 95

Cramer, D. 1997, Basic Statistics for Social Research (London: Routledge)

Dotter, A., Chaboyer, B., Jevremović, D., et al. 2008, ApJS, 178, 89
Ernst, A., Glaschke, P., Fiestas, J., Just, A., \& Spurzem, R. 2007, MNRAS, 377,465

Feltzing, S., Primas, F., \& Johnson, R. A. 2009, A\&A, 493, 913

Ferraro, F. R., Lanzoni, B., Raso, S., et al. 2018, ApJ, 860, 36

Gaia Collaboration, Brown, A. G. A., Vallenari, A., et al. 2018, A\&A, 616, A1

Gaia Collaboration, Prusti, T., de Bruijne, J. H. J., et al. 2016, A\&A, 595, A1

Goldsbury, R., Richer, H. B., Anderson, J., et al. 2010, AJ, 140, 1830

Harris, W. E. 1996, AJ, 112, 1487

Heyl, J., Caiazzo, I., Richer, H., et al. 2017, ApJ, 850, 186

Hong, J., Patel, S., Vesperini, E., Webb, J. J., \& Dalessandro, E. 2019, MNRAS, 483, 2592

Hong, J., Vesperini, E., Sollima, A., et al. 2016, MNRAS, 457, 4507

Libralato, M., Bellini, A., van der Marel, R. P., et al. 2018, ApJ, 861, 99

Milone, A. P., Marino, A. F., Mastrobuono-Battisti, A., \& Lagioia, E. P. 2018, MNRAS, 479, 5005

Milone, A. P., Piotto, G., Bedin, L. R., et al. 2012, A\&A, 540, A16

Milone, A. P., Piotto, G., Renzini, A., et al. 2017, MNRAS, 464, 3636

Nardiello, D., Libralato, M., Piotto, G., et al. 2018, MNRAS, 481, 3382

Nardiello, D., Piotto, G., Milone, A. P., et al. 2015, MNRAS, 451, 312

Piotto, G., Milone, A. P., Bedin, L. R., et al. 2015, AJ, 149, 91

Richer, H. B., Heyl, J., Anderson, J., et al. 2013, ApJL, 771, L15

Tiongco, M. A., Vesperini, E., \& Varri, A. L. 2016, MNRAS, 455, 3693

Tiongco, M. A., Vesperini, E., \& Varri, A. L. 2017, MNRAS, 469, 683

Trenti, M., \& van der Marel, R. 2013, MNRAS, 435, 3272

van der Marel, R. P., \& Anderson, J. 2010, ApJ, 710, 1063

van der Marel, R. P., \& Franx, M. 1993, ApJ, 407, 525

Vesperini, E., McMillan, S. L. W., D'Antona, F., \& D'Ercole, A. 2013, MNRAS, 429, 1913

Wagner-Kaiser, R., Sarajedini, A., von Hippel, T., et al. 2017, MNRAS, 468, 1038

Webb, J. J., \& Vesperini, E. 2017, MNRAS, 464, 1977 